\title{
Responsabilidad social y desarrollo personal: el valor de la práctica temprana
}

\author{
María José Labra Navarro*
}

\begin{abstract}
RESUMEN
El presente artículo presenta los resultados de una investigación desarrollada en el marco del proceso de sistematización de los proyectos sociales elaborados y ejecutados por los estudiantes de la asignatura de Desarrollo Personal perteneciente al Plan Común de la Universidad Católica Silva Henríquez. Esta experiencia formativa busca profundizar y poner en práctica el sello identitario, basado en el respeto, la solidaridad y la búsqueda del bien común, declarado por la Universidad, incentivando el ejercicio de la competencia de responsabilidad social.

De este modo, mediante un enfoque cualitativo y un análisis de contenidos se buscó categorizar y describir los principales aprendizajes relevados por los estudiantes participantes de este proceso, con la finalidad de recoger elementos que permitan la validación y utilización de esta metodología como una herramienta viable de apropiación inicial en los estudiantes de dicha competencia.
\end{abstract}

Palabras Clave: Responsabilidad social - Competencias - Formación Desarrollo personal - Acción social.

\section{Responsabilidade social e desenvolvimento pessoal: o valor da prática temprana}

RESUMO

O presente artigo apresenta os resultados de uma investigação que se desenvolve no marco do processo de sistematização dos projetos sociais elaborados e executados pelos estudantes da aula de Desenvolvimento Pessoal que pertence ao Plano Comum da Faculdade Católica Silva Henríquez. Esta experiência formativa procura aprofundar e colocar em prática o selo identitário, baseando-se no respeito, na solidariedade e procura do bem comum declarado pela Faculdade, incentivando o exercício da competência de responsabilidade social. Deste modo, mediante um enfoque cualitativo e uma análise de conteúdos se procurou categorizar e descrever as principais aprendizagens relevadas pelos estudantes participantes deste processo, com a finalidade de recolher elementos que

* Chilena. Trabajadora Social. Magíster en Participación y Políticas Locales. Docente Escuela de Trabajo Social y Plan Común de la Universidad Católica Cardenal Silva Henríquez. Correo electrónico: mlabra@ucsh.cl 
permitam a validação e utilização desta metodologia como uma ferramenta viável de apropriação inicial nos estudantes de dita competência.

Palavras Chave: Responsabilidade social - Competências - Formação Desenvolvimento Pessoal - Ação social.

\section{Social responsibility and personal development: the value of early practicum}

ABSTRACT

This article presents the results of a study carried out in the framework of the systematization of social projects developed and implemented by the students of the course: Personal Development pertaining to study plan of Universidad Católica Silva Henríquez. This educational experience seeks to strengthen the institutional identity seal, based on respect, solidarity and the common good, declared by the University, encouraging the exercise of the ability of social responsibility. Thus, by using a qualitative approach and content analysis, the study aims to categorize and describe the main learning dimensions learned by the students of this course with a view to collecting information required for the validation and use of this methodology as a didactic tool to help students to develop such competency.

Keywords: Social Responsibility - Competencies - Education - Personal Development - Social Action

\section{Antecedentes}

Hoy en día el concepto de "ser socialmente responsable comienza a ser requisito indispensable en el ámbito educativo. Los futuros profesionales socialmente responsables que con gran interés demanda nuestra sociedad, están hoy y ahora formándose en las aulas de nuestra Universidad" (Carmen de la Calle Maldonado de Guevara, 2007). Sin embargo, debido a la novedad de este tema, todavía hay mucho por definir y concretar en cuanto a qué se entiende por un "universitario comprometido" en el campo social. En la última década del siglo XX hemos sido testigos de un despegue y desarrollo vertiginoso del voluntariado, acompañado por un número creciente de ONG, fundaciones, instituciones gubernamentales, privadas y universidades que entregan servicios a la comunidad y a aquellos sectores de la población más carenciados. "Por otro lado, cada vez son más los centros de formación que incluyen entre sus actividades extraacadémicas algún tipo de acción solidaria: campañas de sensibilización social, proyectos de participación social, experiencias de aprendizaje servicio 
y asignaturas optativas relacionadas con la temática de la solidaridad" (Carmen de la Calle Maldonado de Guevara, 2007). En este contexto, la propuesta educativa que ha realizado la Universidad Católica Silva Henríquez, desde sus inicios ha estado ligada al ámbito de la acción y el compromiso social, figura indiscutible en la apropiación de este sello ha sido su inspirador, el Cardenal Raúl Silva Henríquez ${ }^{1}$, referente universal en la defensa de la vida y el respeto por los Derechos Humanos.

Siguiendo con esta línea de desarrollo, la Universidad Católica Silva Henríquez figura como un proyecto pionero al integrar en sus programas de formación las temáticas del Desarrollo Personal y el Proyecto de Vida con una perspectiva socialmente responsable, respondiendo de este modo al desarrollo del componente de formación personal. Para llevar a cabo este proceso, se ha diseñado un conjunto de actividades curriculares orientadas a la formación valórica del estudiante, las que complementan su formación personal, de acuerdo a sus propios intereses. Este componente se implementa a través de las actividades curriculares comunes para todos los estudiantes y por las actividades electivas. De este modo la unidad académica que unifica todas estas asignaturas es el Plan Común Universidad, integrado por cuatro asignaturas optativas, dos de ellas correspondientes a Formación Teológica, una de Desarrollo Personal y otra a Formación Ética. En esta lógica, surge la idea de incorporar la metodología de proyecto social, la que se intenciona como un espacio de formación práctica inicial de encuentro con la realidad social y personal, que permite ejercitar inicialmente en los estudiantes la competencia de responsabilidad social.

\section{Metodología de proyectos sociales y desarrollo personal}

La metodología de proyectos sociales, intentando responder a este tipo de formación universitaria, se aplica hace aproximadamente dos años desde la asignatura de Desarrollo

1 Destacado sacerdote salesiano de nacionalidad chilena. Las Naciones Unidas reconoció su destacada acción social y humana confiriéndole el Premio Derechos Humanos en el año 1978 y, posteriormente, por la misma razón, recibió el Premio Fundación Bruno Kreysky en Viena el 19 de octubre de 1979. Creó la Vicaría de la Solidaridad, principal organismo defensor de los derechos humanos durante el régimen militar en Chile. Fue precursor del desarrollo de la Universidad Católica Blas Cañas, hoy en su honor, Católica Silva Henríquez, con la opción de brindar preferentemente a jóvenes e hijos de trabajadores de los sectores más desfavorecidos del país una educación de calidad. 
Personal, con la finalidad que mediante la elaboración y ejecución de un proyecto social, los estudiantes se acerquen al encuentro y reconocimiento de un otro, muchas veces fuera de sus aulas, y se comprometan con la realización de distintas actividades en beneficio de la comunidad intra y extra universitaria. Orientados por este propósito los proyectos sociales tienen como principal objetivo que los estudiantes realicen un proceso de asimilación de los contenidos revisados durante el semestre, para luego aplicarlos al servicio de grupos o comunidades ejecutando un proyecto social que dé solución a una necesidad previamente identificada. "En este contexto el ejercicio inicial de la responsabilidad social significa la movilización de conocimientos, recursos humanos, materiales, económicos y redes de relaciones personales" (Iparraguirre, 2008), transformándose en una "oportunidad de probar, en situaciones concretas, el grado de eficiencia de los futuros profesionales y le permite, sobre la base de estas experiencias, actualizar el currículum y sus técnicas según las exigencias del contexto (Iparraguirre, 2008)". Es importante destacar que las asignaturas de Desarrollo Personal abordan las temáticas de: Afectividad y sexualidad; comunicación efectiva; Derechos Humanos y formación ciudadana; Liderazgo y Trabajo en equipo; y Prevención, Autocuidado y calidad de Vida. Considerando estas temáticas, cada asignatura presenta un proyecto en una de las tres modalidades disponibles: Aula (Proyectos que buscan satisfacer una necesidad o problemática dentro del mismo grupo-curso). Pares/universidad (Proyectos desarrollados en el espacio de la universidad y que tienen como beneficiarios a un grupo o el conjunto de la comunidad universitaria); y comunitario (proyectos desarrollados fuera de la universidad y que tienen como beneficiarios escuelas, hogares de niños, jardines infantiles, hogares de ancianos, etcétera). El hecho de que las asignaturas de Desarrollo Personal se enmarquen dentro del Plan Común Universidad, significa que los cursos tienen una orientación transversal, por tanto están constituidos por estudiantes de todas las carreras que imparte la Universidad, siendo esta una característica relevante pues para un alto porcentaje, el realizar en conjunto con sus compañeros un proyecto social, resulta ser su primera experiencia de ayuda directa con carácter multidisciplinario, con el impacto a nivel formativo y personal que eso conlleva, mientras que para otros, con formación anterior en temas relacionados con voluntariado o grupos de acción social, es la oportunidad de poner en práctica habilidades, destrezas y talentos. 
En ese sentido, y luego del cúmulo de experiencias docentes producto de la aplicación y ejecución de la metodología de proyectos sociales, ha parecido interesante para la discusión académica, investigar cuáles serían los aprendizajes relevados por los estudiantes a partir de su desarrollo, identificando si su utilización les permite el desarrollo de la autonomía y el protagonismo en el marco de una educación superior transversal, ampliando su sentido y asociando su uso no solo a una actividad curricular particular, sino que al conjunto de actividades desarrolladas en el proceso de formación. Es importante destacar que los proyectos analizados se elaboraron y ejecutaron entre los meses de octubre y diciembre del año 2011 , y sus resultados fueron presentados por los estudiantes a la comunidad universitaria mediante la realización de la jornada de ciudadanía responsable².

\section{Nociones sobre el concepto de competencia de responsabilidad social}

El concepto de competencia desde el Modelo de Formación ${ }^{3}$ de la Universidad Católica Silva Henríquez, se define como: "aquel encuentro de saberes, no solo pragmáticos y orientados a la producción, sino que aquellos que articulan una concepción del ser, del saber, del saber hacer $y$ de saber convivir (UCSH, 2010). Por otro lado, la competencia de responsabilidad social es definida como: "Aquella capacidad que contribuye al desarrollo humano integral, a través de la identificación, comprensión y ejercicio en libertad, de las acciones y toma de decisiones, considerando el impacto personal, social y ecológico de ellas (Mejías, 2012)". En este contexto la opción de aplicar la metodología de proyectos sociales se sustenta en una estrategia pedagógica basada en la experiencia solidaria, en la cual estudiantes, docentes y comunidad trabajan juntos. La aplicación de esta metodología se manifiesta en una serie de etapas que van desde:

2 Instancia de reflexión y encuentro cívico que permite dar cuenta del trabajo realizado por los optativos de Desarrollo Personal, especialmente la sistematización de los proyectos sociales, desarrollada el viernes 2 de diciembre del 2011 en el patio de la casa central de la UCSH.

3 El modelo de formación se define como el marco orientador y regulador de la formación humana, cristiana, académica y profesional que imparte la Universidad Católica Silva Henríquez. Estas orientaciones asumen los principios de calidad, identidad y sustentabilidad que la definen y que se derivan tanto de su Misión, Visión y Declaración de Principios, como de los documentos acerca de Políticas e Identidad de las Instituciones Salesianas de Educación Superior (IUS) 


\section{Presentación del proyecto social}

Ejecución y posterior socialización de los resultados del proyecto en la jornada de ciudadanía responsable.

Evaluación y sistematización, con miras a aquellos proyectos que pudiesen tener continuidad en otras instancias universitarias como la Dirección de Asuntos Estudiantiles, el Área de voluntariado o la Pastoral.

\section{Aproximación a los aprendizajes relevados por los estudiantes}

Para alcanzar el objetivo principal de este estudio, centrado en describir y categorizar los aprendizajes relevados por los estudiantes, se diseñó una investigación con enfoque cualitativo, utilizando el análisis de contenidos como método de aproximación a tales aprendizajes. Se trabajó con una muestra de 40 educandos pertenecientes a los optativos de comunicación efectiva; liderazgo y trabajo en equipo; afectividad, sexualidad y prevención; autocuidado y calidad de vida, con quienes se realizaron entrevistas, pautas de evaluación y observación participante.

A partir de la información recabada en la fase de campo se obtuvo una multiplicidad de aprendizajes, los cuales se categorizaron de acuerdo a su recurrencia. Se realizó un análisis categorial simple, seleccionando aquellos discursos manifiestos de carácter más frecuente y significativo en virtud de los objetivos de la investigación. Posterior a lo señalado, se obtuvieron las categorías; luego de esto, se llevó a cabo el análisis transversal, del cual surgen las subcategorías. Todo lo anterior permitió elaborar el cuadro que se presenta a continuación:

\begin{tabular}{|l|l|}
\hline Categoría de aprendizaje & Subcategorías \\
\hline \multirow{2}{*}{$\begin{array}{l}\text { Experiencia práctica } \\
\text { enriquecedora }\end{array}$} & Estilo docente cercano y facilitador de la experiencia \\
\cline { 2 - 2 } & Desarrollo de habilidades y talentos \\
\cline { 2 - 2 } & Trabajo en equipo \\
\cline { 2 - 2 } & Ejercicio de la solidaridad \\
\hline Determinar y jerarquizar & Reconocimiento del otro \\
\cline { 2 - 2 } $\begin{array}{l}\text { problemas mediante el } \\
\text { uso del diagnóstico }\end{array}$ & Intervención desde lo multidisciplinario \\
\cline { 2 - 2 } Entregar valores & Visión crítica reflexiva de la realidad \\
\cline { 2 - 2 } & Creatividad e imaginación \\
\hline Reafirmar la vocación & Descubrirse \\
\hline
\end{tabular}


A continuación se presentan descriptivamente algunos de los discursos de los estudiantes de acuerdo a las categorías y subcategorías anteriormente expuestas:

\section{Categoría experiencia práctica enriquecedora}

La teoría por sí sola no compone un aprendizaje significativo, en muchos de los casos los conocimientos relevados surgen de la unión de la práctica, la teoría y lo significativo de esta experiencia, debido a la diversidad de componentes presentes en su ejecución como realidades, escenarios, contextos y actores. Esto es posible constatar mediante la siguiente frase: "Sí, me sirvió muchísimo, me ayudó a descubrir muchas cosas nuevas tanto en mí como en mi alrededor, abrió mi mente... creo que lo más importante de esta experiencia es que trabajamos los contenidos y los llevamos a la práctica... lejos la mejor experiencia que he tenido en el año" (Estudiante $\mathrm{N}^{\circ} 1$ ). Desde otra perspectiva, lo mismo se refleja en: "Creo que estas salidas a terreno sirven mucho más porque uno aprende mediante la práctica, adquiere la experiencia, práctica desde el principio, que es súper enriquecedora y que facilita socializar con los alumnos." (Estudiante $\left.N^{\circ} 2\right)$.

\section{Subcategoría estilo docente cercano y facilitador de la experiencia:}

Un docente próximo y facilitador de la experiencia provoca una mejor y mayor confianza propiciando y potenciando la libre expresión de opiniones e ideas, los estudiantes tienen la percepción de que los proyectos se construyen colectivamente, con la ayuda de todos y gracias a que los docentes se convierten en facilitadores del proceso, así relevan: "Es bueno que los profes nos enseñen como armar proyectos sociales, así con una "parada" cercana, escuchando lo que los estudiantes proponemos... como futuro profe aprendí que eso es súper importante" (Estudiante $\mathrm{N}^{\circ} 3$ ).

La destreza que presente el docente, será fundamental al momento de traspasar a los estudiantes los contenidos, facilitando la priorización de necesidades, la organización de grupos de avance para la elaboración del diagnóstico, salidas a terreno, trabajo en comisiones, etcétera. En este sentido, su labor se orienta a resolver dudas y permitir que sean los propios estudiantes quienes apliquen los contenidos, traduciéndolos en propuestas concretas. La acción social desplegada con cada 
proyecto, permite a su vez generar procesos de reflexión crítica respecto a las propias decisiones y acciones, y el efecto de ellas en su contexto.

\section{Subcategoría desarrollo de habilidades y talentos:}

Uno de los factores que influye en el aprendizaje de nuevas competencias es el clima que se genera en el espacio de la sala de clases, el que se caracteriza por ser menos estructurado, hecho que privilegia el diálogo ayudando a suscitar la confianza necesaria para ir demostrando habilidades y talentos, desde la toma de conciencia de una realidad social desigual, enfatizando en este proceso la importancia de la responsabilidad social: "A mí me gustó mucho hacer este proyecto social, porque me gusta trabajar con la gente, vi que tengo habilidades para eso, que hay una buena llegada... entonces no sé... me motivan harto las desigualdades que hay hoy en el país, entonces ayudar con un pequeño grano de arena para que eso mejore es súper bueno como ejercicio de la profesión" (Estudiante $N^{\circ} 4$ ).

En estos discursos, se evidencia la importancia y la necesidad manifiesta de los estudiantes de contar con experiencias de práctica temprana: "Como futura profe creo que es súper importante contar con estas experiencias de práctica desde el primer año, porque así uno sabe si tiene dedos pa'l piano o no... es un buen ejercicio para poner a prueba la vocación y tomar decisiones" (Estudiante $\mathrm{N}^{\circ} 5$ ).

Lo mismo también se ilustra en el siguiente relato: "Una no solo se siente bien como futura profesional, también como persona porque da ene alegría cachar que la carrera te gusta y que soy buena, en ese contacto con la gente y que ellos te lo digan y reconozcan" (Estudiante $\mathrm{N}^{\circ} 6$ ).

\section{Subcategoría trabajo en equipo:}

La importancia del trabajo en equipo, referido principalmente a la toma de decisiones colectivas, considerando la opinión de cada uno, buscando el consenso en la designación de las distintas tareas de acuerdo a las destrezas, habilidades y carrera cursada, en un clima de respeto y confianza. Así comentaron: "Se podrían realizar más actividades como la del proyecto ya que desde mi perspectiva sirvió para motivar a participar a la mayoría del curso, además de fomentar el trabajo en equipo, designar comisiones de trabajo y hacer que cada uno se sintiera a gusto y aportando de verdad" (Estudiante $\mathrm{N}^{\circ} 7$ ). 


\section{Subcategoría ejercicio de la solidaridad:}

El percibir la solidaridad como un motor que guía las acciones no solo del proyecto social, sino de su proyecto de vida, permite a los estudiantes una mirada integral, relacionada con el trabajo colaborativo y organizado en pos de cumplir objetivos, que facilite la capacidad de reflexión y ejercicio del rol ciudadano: "Aprendí a cambiar mi modo de ver las cosas, la solidaridad no es caridad, la solidaridad es trabajo organizado con sentido social, tal como lo hacía el Cardenal" (Estudiante $\mathrm{N}^{\circ} 8$ ).

La figura y el legado del Cardenal Raúl Silva Henríquez, es relevada como símbolo de solidaridad que caracteriza la formación profesional que entrega la universidad: "Con esta actividad reflexioné sobre mi proyecto personal, desde la elección profesional y lo que la universidad propone a través del legado del Cardenal especialmente desde los Derechos Humanos y la solidaridad" (Estudiante $N^{\circ}$ 9).

Se releva también la importancia de esta Universidad como un ente promotor de la solidaridad entre la comunidad intra y extrauniversitaria, el ejercicio de la responsabilidad social entonces está estrechamente ligado al carácter solidario y humanizante de las acciones desarrolladas por los estudiantes, en coherencia con los principios que sustentan a la Universidad.

\section{Categoría determinar y jerarquizar problemas}

Un elemento central de este proceso es la realización del diagnóstico, previo a la elaboración del proyecto social; esta herramienta permite reconocer desde el discurso de los actores las necesidades sentidas. Los estudiantes enfatizan que para lograr este propósito es necesario definir y jerarquizar aquello que efectivamente es posible de realizar, para así no generar falsas expectativas o comprometerse en acciones que no están dentro del rango de ejecución del proyecto, para ello es fundamental tener en cuenta elementos importantes como los recursos y el tiempo. Es de gran importancia generar un compromiso responsable con la comunidad o grupo intervenido: "Yo creo que uno cuando va a proponer un proyecto tiene que tener muy claro cuáles son los problemas de esa comunidad... por ejemplo, en nuestro proyecto quizás lo más urgente no era trabajar la autoestima, era pintar la escuela, mejorar los baños... pero los recursos no daban... entonces lo otro que quizás no era tan 
urgente lo tomamos y lo convertimos en algo beneficioso y entretenido para ellos" (Estudiante $\mathrm{N}^{\circ} 10$ ).

Se identifica la importancia del diagnóstico como un aprendizaje transversal a cualquier carrera, relevado como un instrumento eficaz a la hora de acercarse a una problemática: "Para cualquier profesión es importante saber diagnosticar... aquí todos pensamos e ideamos la manera de ayudar y eso es súper relevante hacerlo desde el primer año, por ejemplo para mí que estudio derecho" (Estudiante $\mathrm{N}^{\circ} 11$ ).

\section{Subcategoría reconocimiento del otro:}

Las intervenciones sociales no solo implican conocer una realidad, sino además, involucrarse en las dinámicas cotidianas de los sujetos y reflexionar críticamente sobre aquel sistema muchas veces injusto, que posibilita la existencia de aquellas desigualdades sociales. El reconocer a otro, es reconocer también esa realidad, para aprender a trabajar con y desde el origen de la problemática que le aqueja. Tiene que ver con la relación interpersonal presente en cualquier proceso de comunicación. Este reconocimiento no solo permite dimensionar el contexto social en el que se encuentran inmersos, sino que también le otorga sentido a las acciones realizadas: "No solo pensar en uno mismo sino también en lo que nos rodea, en ese otro que necesita nuestro apoyo, que de una u otra forma aquel problema nos afecta igual como personas, porque nace de un contexto de desigualdad que nos golpea... la realidad está ahí, solo debemos aprender de ella" (Estudiante $\mathrm{N}^{\circ} 12$ ).

Para transformar contextos, es imperante la necesidad de valorar y apreciar las diferencias, por ende es altamente valorada la libertad de elección que los proyectos sociales les brindan, pudiendo enfocar su proceso de acción social hacia aquellos grupos o comunidades que identifican como prioritarias: "Este proyecto me permitió no solo compartir con mis compañeros, sino que también con la comunidad con la que trabajamos, y creo que fue súper importante el diálogo y el respeto, porque uno está en constante interacción con el otro, de construir juntos, y eso nos va dando herramientas" (Estudiante $\mathrm{N}^{\circ} 1$ ).

\section{Subcategoría intervención desde lo multidisciplinario:}

La posibilidad de trabajar de manera multidisciplinaria permite conjugar diversos conocimientos al servicio del proyecto social y de una intervención acorde con las necesidades del contexto. Se evidencia además que para realizar una intervención que sea de 
utilidad es necesario poner en común conocimientos, habilidades y técnicas de trabajo que provengan de las teorías entregadas por las diversas escuelas, donde el desarrollo teórico y práctico es un factor relevante en el quehacer profesional, lo que se evidencia en la siguiente frase: "Tenemos que saber construir proyectos desde elementos prácticos pero también desde elementos teóricos, entonces la riqueza de este proyecto es que se respetan y validan todas esas miradas, que después son las mismas que estarán cuando ejerzamos la profesión de cada uno" (Estudiante $\left.\mathrm{N}^{\circ} 4\right)$.

\section{Subcategoría visión crítica reflexiva de la realidad:}

El éxito de los proyectos depende de la capacidad anterior y posterior a todo el proceso, de analizar crítica y reflexivamente aquellas desigualdades presentes en el sistema con aquellos desafíos que la sociedad del conocimiento impone a los nuevos profesionales: "Este proyecto me ayudó a cambiar mi perspectiva, porque uno ve en el terreno los problemas sociales de la gente, que a veces son tan fáciles de solucionar pero a nadie parece que le interesa, en la escuela los baños estaban súper pencas ${ }^{4}$ y eso era súper malo para los niños, y uno piensa pero cómo si es algo tan básico" (Estudiante $\mathrm{N}^{\circ} 12$ ).

De este modo, los estudiantes se ven enfrentados a nuevos desafíos en el plano de su formación que abarcan ámbitos más personales, de un cuestionamiento que incentive la acción y el cambio social, considerando la importancia de la vinculación con el otro, de comprender su situación y de la importancia de movilizar recursos personales y destrezas colectivas para ayudar a mejorar estas condiciones, por ende también es la posibilidad de ir conformando proyectos de vida en el plano personal y social: "Creo que uno también proyecta en su vida aquel aporte como social que va haciendo, porque igual quiere decir que uno se preocupa, piensa, no sé... como que reflexiona $y$ se hace responsable por su entorno y por ayudar de alguna manera a que las cosas cambien... entonces eso también es reconfortante para la vida de uno... después de lo que hicimos con los niños yo llegué súper contento a mi casa" (Estudiante $\mathrm{N}^{\circ}$ 5).

\section{Categoría entregar valores}

El estudiante reconoce en la transmisión de valores una característica esencial para el desarrollo del proyecto social; se

4 Chilenismo que en este contexto significa en mal estado. 
alude a que las acciones del proyecto debían tender a reforzar valores como la solidaridad, el respeto, la comunicación y el amor, por ende los estudiantes se asumen como portadores de dichos valores y que parte de su proceso de formación reside en saber transmitirlos: "es importante saber que igual que ellos (compañeros de curso), uno está en un cuento de formación, entonces uno también trasmite lo que es y los valores que tiene... a veces hay gente que es súper distinta a uno... pero parte de eso también es saber que esa diversidad de cosas y personas, es buena y que sirve pa' hacer un mejor proyecto" (Estudiante $\mathrm{N}^{\circ}$ 6).

Del mismo modo, este traspaso de estos valores tiene mucha relación con la experiencia de desarrollo personal y la influencia de contexto familiar y social desde donde provenga el estudiante. Relevan de este modo una mirada ética y comprometida, que mezcla esa carga que cada uno porta con aquello que entrega el plan de formación y el proyecto educativo que cada estudiante ha decidido emprender: "Es importante la experiencia de vida de cada uno, de los valores que trae, de cómo se crió y de por qué eligió lo que estudia... no sé, también de lo que quiere entregar en su relación con otro" (Estudiante $\left.\mathrm{N}^{\circ} 8\right)$.

\section{Subcategoría creatividad e imaginación:}

La creatividad consiste en generar ideas nuevas y saber comunicarlas, es visualizada como la energía de llevar a cabo cambios en el entorno cotidiano. Es aquel proceso o facultad que permite hallar relaciones y soluciones novedosas, partiendo de aquello que se detecta en el diagnóstico, pues abarca no solo la posibilidad de solucionar un problema ya detectado, sino que también implica la oportunidad de descubrir un problema allí, donde el resto de las personas no lo ven y no encuentran solución.

Por tanto, esta es relevada como una habilidad que debe estar presente a lo largo de la vida académica y personal: "Yo creo que ser creativo sirve no solo pa'l proyecto social, sirve para la vida" (Estudiante $\left.\mathrm{N}^{\circ} 2\right)$.

Se constituye además, en una herramienta que facilita la búsqueda de soluciones no solo a los problemas personales y académicos, sino que también a aquellos que surjan del contacto y relación con el contexto y la comunidad: "En el proyecto social hay que aplicar toda la creatividad, porque es súper "fome"5 ir a hacer lo mismo

5 Chilenismo utilizado para referirse a una situación sin gracia o aburrida. 
que hacen todos los días con los profes... la idea era hacer algo distinto, que los ayudara a darse cuenta de lo importante del respeto" (Estudiante $\mathrm{N}^{\circ} 5$ ).

\section{Subcategoría habilidades comunicacionales: Cercanía y empatía}

Ser hábiles comunicacionalmente posibilita una mayor y mejor comprensión de los contextos sociales donde se desarrollarán los procesos de intervención, situando a los estudiantes en espacios que requieren de relaciones de cercanía y empatía: "Es bueno el clima de cercanía que se da en la sala, aunque somos todos de distintas carreras nos complementamos bien, nos escuchamos con respeto $y$ cuando alguien proponía algo todos tratábamos de ser empáticos con esas ideas" (Estudiante $\mathrm{N}^{\circ}$ 9).

En este sentido, la sala de clases se convierte en el escenario ideal para debatir y reflexionar respecto a los distintos puntos de vista y miradas en relación a la intervención. Otro aspecto que resaltan los estudiantes, tanto en el espacio de la sala de clases como en la ejecución del proyecto, dice relación con establecer lazos de cercanía con el otro, de ser acogedores, saber escuchar, contener y mantener un constante clima de respeto: "Cuando llegaron los niños a la universidad, y la profesora nos contó un poco de sus historias, fue difícil tratar de imaginar cómo sería vivir sin una familia, me impactó... pero creo que eso mismo me ayudó a acercarme más, conversar. hacerles cariño y tratar de ser empática" (Estudiante $\mathrm{N}^{\circ} 2$ ).

\section{Categoría reafirmar la vocación}

La vocación es aquel deseo entrañable hacia lo que aspiran a convertirse en un futuro, aquello que pretenden realizar por el resto de sus vidas. Sin embargo, reafirmarla y alcanzarla es algo que se determina por la obtención de conocimientos que van nutriendo y encantando la opción profesional. En este sentido, los estudiantes ven en la realización del proyecto social la posibilidad de poner en práctica aquellas destrezas propias de la elección profesional: "Igual es heavy cuando hacemos el proyecto y llegamos al lugar... porque igual si es un trabajo con mujeres, o con niños y te das cuenta que eso no te gusta o que no tienes la habilidad, igual es complicado" (Estudiante $\mathrm{N}^{\circ} 6$ ). Ello también se grafica en la siguiente frase: "Con esto reafirmé que elegí bien mi carrera y que tengo vocación para el trabajo social" (Estudiante $\mathrm{N}^{\circ} 1$ ). 
La vocación se constituye entonces en un medio para alcanzar la autorrealización y la transformación personal y social. Valoran las herramientas teóricas y prácticas entregadas en el desarrollo de la metodología de proyectos sociales, considerándolas de gran utilidad en el camino de su formación, ayudándoles justamente a descubrir y encontrar, desde la ejecución de esta herramienta, teórico-práctica inicial, elementos para ampliar su mirada y trascender.

\section{Conclusiones}

Quisiera partir reflexionando en torno a la historia detrás de este proceso. La Universidad Católica Silva Henríquez es una institución pionera al incluir dentro de su modelo de formación la temática de la Formación Personal, opción que busca dar respuesta a un estilo de enseñanza inspirado en la identidad salesiana, y que tiene como fin la formación integral de los estudiantes, de cara al compromiso institucional con una sociedad más justa y equitativa. En este sentido, es de suma importancia el aporte y la experiencia tanto práctica, como académica que el equipo docente a cargo del componente de formación personal ha venido desarrollando y que ha permitido la inclusión, entre otras herramientas, de la metodología de proyectos sociales como un recurso pedagógico didáctico y eficaz. No en vano son los mismos estudiantes quienes, mediante sus discursos, han valorado y resaltado el impacto que este estilo de enseñanza posee, al permitirles desarrollar desde la valoración de su proyecto de vida, una experiencia práctica inicial de encuentro y reconocimiento con un otro.

Cuando se habla de responsabilidad social, se ha intentado mostrar lo fundamental que es considerar esta dimensión en los procesos educativos, investigativos, sociales y de gestión que experimenta esta Universidad. Para lograr este propósito, es de vital importancia que se genere una integración curricular que responda a esta lógica, intentando hacer congruentes con ella los programas formativos, mediante la elección de técnicas, metodologías y estrategias educativas afines, que permitan insertar armónicamente la formación en responsabilidad, de este modo el desafío a futuro estaría centrado en la labor de potenciar dicha competencia a través de su seguimiento, sistematización y articulación, con otras actividades curriculares, intencionando que la metodología de proyectos sociales se utilice no solo desde las asignaturas de desarrollo personal, sino que también 
su aplicación se amplíe a las de ética, teología y otras actividades formativas complementarias como son los talleres de la Dirección de Asuntos Estudiantiles, los electivos de formación general, el voluntariado de la dirección de pastoral, entre otros. Este hecho, sin lugar a dudas permitirá ampliar su perspectiva y entenderse no solo como un esfuerzo que depende en sí mismo de una actividad, sino que puede y debe ser aplicable con esta perspectiva diversa e inclusiva.

Desde la asignatura de Desarrollo Personal, se ha buscado formar estudiantes en el ejercicio de la competencia de responsabilidad social, posibilitando el ejercicio de un análisis social más crítico y reflexivo, de la mano del desarrollo de experiencias de vinculación con el medio, que se configuran como una demanda creciente en nuestro panorama universitario. En relación a esta experiencia, sería posible inferir que los estudiantes logran ir más allá de la obligatoriedad de la calificación que impone la ejecución del proyecto, comprendiendo este proceso desde una mirada integral, relacionada con el trabajo colaborativo y organizado en torno a cumplir objetivos, generándose de este modo, una estrecha relación entre afectividad, autoestima, habilidades interpersonales y personales, habilidades comunicacionales, proyección, historia y sobre todo valores. Se suma a esto, el desarrollo de un trabajo interdisciplinario, lo que supone la confluencia de distintas miradas, hecho que posibilita un mejor y mayor alcance de los proyectos. Es también gracias a este proceso, que ejercitan su vocación de servicio, hecho que amplía la comprensión de su carrera reforzando su sentido y expandiendo sus posibilidades.

En el esfuerzo por sacar adelante el proyecto social que cada curso y que cada estudiante ha diseñado, es necesaria su voluntad, su compromiso y motivación por aprender, asimilando con claridad la utilidad y el beneficio que esta experiencia educativa implica para su propia vida y para su desarrollo, proceso que puede ser vivido con mayor o menor intensidad, pero que sin duda, supone un descubrimiento personal importante y significativo. Así, es posible inferir que cuando un estudiante logra actuar, desempeñarse de diferentes formas sobre su realidad y solucionar problemas, interactuar eficazmente con otros, enfrentar situaciones complejas y buscar soluciones, cuando aumenta su deseo de comprender al otro, de ponerse en su lugar, de intercambiar ideas, sentimientos, anhelos y sueños, es porque en definitiva está experimentando aquello que le une a un otro, 
que es mucho más grande que aquello que lo diferencia y que marcará en muchos sentidos su experiencia universitaria.

\section{Referencias bibliográficas}

De la Calle, C.; Guevara, J.; Giménez, P. (2007). La formación de la Responsabilidad Social en la Universidad. Revista Complutense de Educación, Vol. 18, N².

Universidad Católica Silva Henríquez (2010). Documento de Apoyo a la Gestión Curricular. Santiago de Chile. UCSH.

Iparraguirre, A. (2008). La Responsabilidad Social de la Universidad en la Promoción del Capital Social para el Desarrollo Sustentable. Recuperado en abril de 2012. Disponible en http://www.eumed.net

Mejías, C. (2012). Competencias; epistemología, paradigma y metodología: Hacia la construcción y graduación de las competencias. Sello Universidad Católica Silva Henríquez. Santiago de Chile.UCSH.

SILVA, R. (1982). La Universidad Católica; Su razón de ser. En: "El Cardenal nos ha Dicho". Miguel Ortega (Comp). Santiago de Chile. Salesiana. 